\title{
$n$-color overpartitions, lattice paths, and multiple basic hypergeometric series
}

\author{
Olivier Mallet ${ }^{\dagger}$ \\ LIAFA, Université Paris Diderot - Paris 7, Case 7014, F-75205 Paris Cedex 13, France
}

\begin{abstract}
We define two classes of multiple basic hypergeometric series $V_{k, t}(a, q)$ and $W_{k, t}(a, q)$ which generalize multiple series studied by Agarwal, Andrews, and Bressoud. We show how to interpret these series as generating functions for special restricted lattice paths and for $n$-color overpartitions with weighted difference conditions. We also point out that some specializations of our series can be written as infinite products, which leads to combinatorial identities linking $n$-color overpartitions with ordinary partitions or overpartitions.

Résumé. Nous définissons deux classes de séries hypergéométriques basiques multiples $V_{k, t}(a, q)$ et $W_{k, t}(a, q)$ qui généralisent des séries multiples étudiées par Agarwal, Andrews et Bressoud. Nous montrons comment interpréter ces séries comme les fonctions génératrices de chemins avec certaines restrictions et de surpartitions $n$-colorées vérifiant des conditions de différences pondérées. Nous remarquons aussi que certaines spécialisations de nos séries peuvent s'écrire comme des produits infinis, ce qui conduit à des identités combinatoires reliant les surpartitions $n$-colorées aux partitions ou surpartitions ordinaires.
\end{abstract}

Keywords: $n$-color overpartitions, lattice paths, multiple series

\section{Introduction}

Many multiple series linked to partitions and related objects have been discovered, such as Andrews' generalization of the Rogers-Ramanujan identities (8) or an infinite family which was studied a few years ago in (6). The purpose of this paper is to study classes of multiples series which generalize the multiple series appearing in (3, Section 5) as well as generating functions from (19) related to $n$-color overpartitions.

The multiple series we will study are the following:

$$
V_{k, t}(a, q)=\sum_{n_{1} \geq n_{2} \geq \cdots \geq n_{r} \geq 0} \frac{(-1 / a)_{n_{1}} a^{n_{1}} q^{\left(\begin{array}{c}
n_{1}+1 \\
2
\end{array}\right)+n_{2}^{2}+\cdots+n_{r}^{2}-n_{1}-\cdots-n_{t}+\left(\begin{array}{c}
n_{r} \\
2
\end{array}\right) \chi(k \text { is even })\left(1-q^{n_{t}}\right)}}{(q)_{n_{1}-n_{2}} \cdots(q)_{n_{r-1}-n_{r}}(q)_{n_{r}}\left(q ; q^{2}\right)_{n_{r}}}
$$

\footnotetext{
${ }^{\dagger}$ The author is partially supported by the ANR project GAMMA.
} 
where $k \geq 2, r=\left\lfloor\frac{k}{2}\right\rfloor, 0 \leq t \leq r$, and $q^{-n_{1}-\cdots-n_{t}}\left(1-q^{n_{t}}\right)$ is taken to be 1 if $t=0$, and

$$
W_{k, t}(a, q)=\sum_{n_{1} \geq n_{2} \geq \cdots \geq n_{r} \geq 0} \frac{(-1 / a)_{n_{1}} a^{n_{1}} q^{\left(\begin{array}{c}
n_{1}+1 \\
2
\end{array}\right)+n_{2}^{2}+\cdots+n_{r}^{2}+n_{1}+\cdots+n_{r}+n_{k-t}+\cdots+n_{r}+\left(\begin{array}{c}
n_{r} \\
2
\end{array}\right) \chi(k \text { is even })}}{(q)_{n_{1}-n_{2}} \cdots(q)_{n_{r-1}-n_{r}}(q)_{n_{r}}\left(q ; q^{2}\right)_{n_{r+1}}}
$$

where $k \geq 2, r=\left\lfloor\frac{k}{2}\right\rfloor$, and $k-r-1 \leq t \leq k-1$.

Here we have employed the standard $q$-series notation (15):

$$
(a)_{n}=(a ; q)_{n}=\prod_{j=0}^{n-1}\left(1-a q^{j}\right)
$$

and

$$
\left(a_{1}, a_{2}, \ldots, a_{k} ; q\right)_{n}=\left(a_{1} ; q\right)_{n}\left(a_{2} ; q\right)_{n} \cdots\left(a_{k} ; q\right)_{n} .
$$

The special case $a \rightarrow 0$ of these series was studied analytically in (3); it was interpreted combinatorially in (2) using $n$-color partitions and in (4) using lattice paths. The instances $V_{2,0}(1, q), V_{3,0}(1, q)$, $W_{2,1}\left(q^{-1}, q\right)$, and $W_{3,1}\left(q^{-1}, q\right)$ were interpreted combinatorially in (19) using $n$-color overpartitions, which extend the $n$-color partitions studied in the 1980's by Agarwal, Andrews and Bressoud (see (5) and references therein) based on work of Baxter (2, Section 3); furthermore, $V_{3,0}(a ; q)$ was interpreted in terms of lattice paths and Frobenius symbols by Corteel (12). An $n$-color partition is a partition where a part $n$ can appear in $n$ colors denoted by subscripts: $n_{1}, n_{2}, \ldots, n_{n}$ and the parts are ordered first by size and then by color. Now, an $n$-color overpartition (19) is an $n$-color partition in which the final occurrence of a part $n_{j}$ may be overlined. For example, the six $n$-color overpartitions of 2 are

$$
(2,2),(2,1),\left(\overline{2}_{2}\right),\left(\overline{2}_{1}\right),\left(1_{1}, 1_{1}, 1_{1}\right),\left(1_{1}, 1_{1}, \overline{1}_{1}\right) .
$$

For any integer $t \geq 0$, we can define similarly $(n+t)$-color partitions (3) and overpartitions, where the possible parts of size $n$ are $n_{1}, n_{2}, \ldots, n_{n+t}$; note that if $t>0$, an $(n+t)$-color partition or overpartition can contain a part of size 0 .

The following theorems are the combinatorial interpretations of $V_{k, t}(a, q)$ and $W_{k, t}(a, q)$ using $(n+t)$ color overpartitions:

Theorem 1.1 $V_{k, t}(a, q)$ is the generating function for $(n+t)$-color overpartitions such that

(i) for all parts $m_{i}$ and $n_{j}$ such that $m \geq n$, if $\left(\left(m_{i}-n_{j}\right)\right)$ plus the number of overlined parts whose size lies in the interval $[n, m)$ is nonpositive, then it is even and greater than or equal to $-2 \min (i-1, j-1, k-3)$, and

(ii) ift $\geq 1$, there is a part of the form $x_{x+t}$,

where the exponent of $q$ counts the size and that of a counts the number of overlined parts.

Theorem 1.2 $W_{k, t}(a, q)$ is the generating function for $(n+t)$-color overpartitions counted by $V_{k, t}(a, q)$ such that no part of the form $x_{x+t}$ is overlined.

Another combinatorial interpretation of these multiple series uses a family of lattice paths that appeared in (14) to interpret a generalization of the Andrews-Gordon identities to overpartitions: 
Theorem 1.3 $V_{k, t}(a, q)$ is the generating function for lattice paths which start at $(0, t)$ and have no valley above height $k-3$ (or no valley at all if $k=2$ ) where the exponent of $q$ counts the major index and that of a counts the number of South steps.

Theorem 1.4 $W_{k, t}(a, q)$ is the generating function for lattice paths counted by $V_{k, t}(a, q)$ such that the first peak is a NESE peak if its coordinates are of the form $(x, x+t)$.

We remark that if there are no overlined parts or no South steps, which corresponds to the case $a \rightarrow 0$, we recover the combinatorial interpretations of the multiple series from (3).

Finally, the previously mentioned specializations of our multiple series were shown to be infinite products, which implies several identities linking $n$-color (over)partitions with their ordinary counterparts. We have found other such identities of the Rogers-Ramanujan type, which will be discussed at the end of this paper:

Theorem 1.5 We have

$$
\begin{aligned}
V_{k, 0}(1, q) & =\frac{(-q)_{\infty}\left(q^{2 k-1} ; q^{2 k-1}\right)_{\infty}}{(q)_{\infty}\left(-q^{2 k-1} ; q^{2 k-1}\right)_{\infty}} \\
V_{k, 0}\left(q^{-1}, q^{2}\right) & =\frac{\left(q^{2} ; q^{4}\right)_{\infty}\left(q^{4 k-4}, q^{4 k}, q^{8 k-4} ; q^{8 k-4}\right)_{\infty}}{(q)_{\infty}} \\
W_{k, t}\left(q^{-1}, q\right) & =\frac{(-q)_{\infty}\left(q^{2 k-1-2 t}, q^{2 k-1+2 t}, q^{4 k-2} ; q^{4 k-2}\right)_{\infty}}{(q)_{\infty}} .
\end{aligned}
$$

Each of these identities can be interpreted combinatorially. Here, we will just state the interpretation of (1.5).

Corollary 1.6 Let $A_{k}(\ell)$ be the number of n-color overpartitions of $\ell$ such that for all parts $m_{i}$ and $n_{j}$ satisfying $m \geq n$, if $\left(\left(m_{i}-n_{j}\right)\right)$ plus the number of overlined parts whose size lies in the interval $[n, m)$ is nonpositive, then it is even and greater than or equal to $-2 \min (i-1, j-1, k-3)$. Let $B_{k}(\ell)$ be the number of overpartitions into parts not divisible by $2 k-1$. Then $A_{k}(\ell)=B_{k}(\ell)$.

For instance, we have that $A_{3}(4)=B_{3}(4)=14$. The $n$-color overpartitions counted by $A_{3}(4)$ are

$$
\left(4_{4}\right),\left(4_{3}\right),\left(4_{2}\right),\left(4_{1}\right),\left(\overline{4}_{4}\right),\left(\overline{4}_{3}\right),\left(\overline{4}_{2}\right),\left(\overline{4}_{1}\right),\left(3_{1}, 1_{1}\right),\left(\overline{3}_{1}, 1_{1}\right),\left(3_{1}, \overline{1}_{1}\right),\left(\overline{3}_{1}, \overline{1}_{1}\right),\left(3_{2}, \overline{1}_{1}\right),\left(\overline{3}_{2}, \overline{1}_{1}\right)
$$

and the overpartitions counted by $B_{3}(4)$ are

$$
\begin{gathered}
(4),(\overline{4}),(3,1),(\overline{3}, 1),(3, \overline{1}),(\overline{3}, \overline{1}),(2,2),(2, \overline{2}),(2,1,1), \\
(\overline{2}, 1,1),(2,1, \overline{1}),(\overline{2}, 1, \overline{1}),(1,1,1,1),(1,1,1, \overline{1}) .
\end{gathered}
$$

The paper is organized as follows. In Section 2, we give the necessary definitions on lattice paths and prove Theorems 1.3 and 1.4. In Section 3, we study the interpretation in terms of $n$-color overpartitions proving Theorems 1.1 and 1.2. In Section 4, we prove Theorem 1.5 using the Bailey lattice structure from (3). We conclude in Section 5 with ideas for future research. 


\section{Lattice paths}

The multiple series from (3) were interpreted in (4) using lattice paths introduced in (9). In this section, we will interpret our multiple series using a generalized family of lattice paths introduced by Corteel and the author in (14). The proofs will be very similar to that of Proposition 1.4 of (4).

We study paths in the first quadrant that start on the $y$-axis, end on the $x$-axis, and use four kinds of unitary steps :

- North-East $N E:(x, y) \rightarrow(x+1, y+1)$,

- South-East $S E:(x, y) \rightarrow(x+1, y-1)$,

- South $S:(x, y) \rightarrow(x, y-1)$,

- East $E:(x, 0) \rightarrow(x+1,0)$.

The height corresponds to the $y$-coordinate. A South step can only appear after a North-East step or at the beginning of the path and an East step can only appear at height 0 . A peak is a vertex preceded by a North-East step (or located at the beginning of the peak) and followed by a South step (in which case we call it a NES peak) or by a South-East step (in which case we call it a NESE peak). A valley is a vertex preceded by a South or South-East step and followed by a North-East step. A mountain is a portion of the path that starts at the beginning of the path or at height 0 , stays above the $x$-axis, and ends at height 0 . The major index of a path is the sum of the abscissas of its peaks. See Figure 1 for an example.

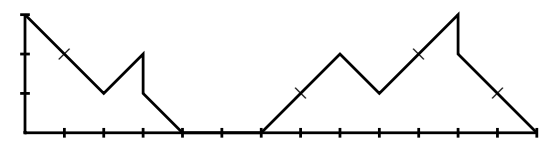

Fig. 1: This path has four peaks: two NESE peaks (located at $(0,3)$ and $(8,2)$ ) and two NES peaks (located at $(3,2)$ and $(11,3))$. There are two valleys, located at $(2,1)$ and $(9,1)$, and two mountains. The major index is $0+3+8+11=22$.

Proof of Theorem 1.3: We first consider the case $r=1$. We have that

$$
V_{3,0}(a, q)=\sum_{n \geq 0} \frac{(-1 / a)_{n} a^{n} q^{\left(\begin{array}{c}
n+1 \\
2
\end{array}\right)}}{(q)_{n}\left(q ; q^{2}\right)_{n}} .
$$

The factor $a^{n} q^{\left(\begin{array}{c}n+1 \\ 2\end{array}\right)}$ generates the path of $n$ NES peaks starting at $(0,0)$ and ending at $(n, 0)$. The factor $(-1 / a)_{n}$ generates a partition $\delta$ into distinct parts which lie between 0 and $n-1$, which is encoded by transforming the $i$ th peak from the right into a NESE peak if there is a part $i-1$ in $\delta$ for $1 \leq i \leq n$. The factor $(q)_{n}^{-1}$ generates a partition $\lambda$ into $n$ nonnegative parts, which is encoded by inserting $\lambda_{n}$ East steps before the leftmost mountain and $\lambda_{i}-\lambda_{i+1}$ East steps before the $i$ th mountain from the right for $1 \leq i<n$. The factor $\left(q ; q^{2}\right)_{n}^{-1}$ generates a partition $\mu$ into odd parts which lie between 1 and $2 n-1$. This partition is encoded by letting the $i$ th peak from the right grow to height $f_{2 i-1}(\mu)+1$ (where $f_{\ell}(\mu)$ is the multiplicity of $\ell$ in $\mu$ ) for $1 \leq i \leq n$. This procedure generates all paths satisfying the conditions 
of the theorem for $k=3$ and $t=0$ and it is uniquely reversible, so Theorem 1.3 is proved for $k=3$ and $t=0$.

The case $k=2$ and $t=0$ is the same except that we have an additional factor $q^{\left(\begin{array}{c}n \\ 2\end{array}\right)}$ which inserts an East step between each of the peaks so that there are no valleys.

For the case $k=3$ and $t=1$, we have

$$
V_{3,1}(a, q)=\sum_{n \geq 0} \frac{(-1 / a)_{n} a^{n} q^{\left(\begin{array}{c}
n+1 \\
2
\end{array}\right)-n}\left(1-q^{n}\right)}{(q)_{n}\left(q ; q^{2}\right)_{n}}=\sum_{n \geq 0} \frac{(-1 / a)_{n} a^{n} q^{\left(\begin{array}{c}
n+1 \\
2
\end{array}\right)-n}}{(q)_{n}\left(q ; q^{2}\right)_{n}}-\sum_{n \geq 0} \frac{(-1 / a)_{n} a^{n} q^{\left(\begin{array}{c}
n+1 \\
2
\end{array}\right)}}{(q)_{n}\left(q ; q^{2}\right)_{n}} .
$$

Remember that

$$
\frac{(-1 / a)_{n} a^{n} q^{\left(\begin{array}{c}
n+1 \\
2
\end{array}\right)}}{(q)_{n}\left(q ; q^{2}\right)_{n}}
$$

is the generating function for paths with $n$ peaks starting at $(0,0)$ with no valleys above height 0 . Introducing the factor $q^{-n}$ corresponds to removing the first step of such a path; when we do so, we can obtain a path with $n$ peaks starting at $(0,0)$, if that first step was an East step, or a path with $n$ peaks starting at $(0,1)$, if the first peak was a North-East step. Since the paths we want to count are those from the second case, the subtraction in (2.2) gives us the right generating function.

The case $k=2$ and $i=1$ is identical except that, again, we have at least an East step between each of the peaks.

The proof is completed by induction. We first need to recall the definition of the relative height of a peak. This notion was defined by Bressoud in (11) for the paths with no South steps and a simpler version was given by Berkovich and Paule in (10). Their definition was adapted in (14) for the paths with South steps.

Definition $2.1((14))$ The relative height of a peak $(x, y)$ is the largest integer $h$ for which we can find two vertices on the path, $\left(x^{\prime}, y-h\right)$ and $\left(x^{\prime \prime}, y-h\right)$, such that $x^{\prime}<x \leq x^{\prime \prime}$ and such that between these two vertices there are no peaks of height $>y$ and every peak of height $y$ has abscissa $\geq x$.

Due to space constraints, we will only treat the case $t=0$, since the case $t>0$ is quite similar. We will need to use the following lemma from (4):

Lemma $2.2((4))$

$$
\frac{q^{n_{1}^{2}+\cdots+n_{r}^{2}}}{(q)_{n_{1}-n_{2}} \cdots(q)_{n_{r-1}-n_{r}}(q)_{n_{r}}\left(q ; q^{2}\right)_{n_{r}}}
$$

is the generating function for paths with no South steps starting at $(0,0)$, with no valleys above height $k-3$, and such that for $1 \leq j \leq r$, there are exactly $n_{j}$ peaks whose relative height is at least $j$ and for which the difference between the height and the relative height is at most $k-2 j-1$.

We consider a path with no South steps starting at $(0,0)$, with no valleys above height $k-5$, and such that for $2 \leq j \leq r$, there are $n_{j}$ peaks whose relative height is at least $j-1$ and for which the difference between the height and the relative height is at most $k-2 j-1$. By Lemma 2.2, such paths are counted by

$$
\frac{q^{n_{2}^{2}+\cdots+n_{r}^{2}}}{(q)_{n_{2}-n_{3}} \cdots(q)_{n_{r-1}-n_{r}}(q)_{n_{r}}\left(q ; q^{2}\right)_{n_{r}}}
$$


The factor $\left.(-1 / a)_{n_{1}} a^{n_{1}} q^{\left({ }^{n_{1}+1}\right)}\right) /(q)_{n_{1}-n_{2}}$ is introduced by a procedure inspired by (4) and (14).

We first perform a "volcanic uplift" by inserting at each peak a North-East step followed by a South step. This operation introduces a factor $a^{n_{2}}$ and increases the major index of the path by

$$
1+2+\cdots+n_{2}=\left(\begin{array}{c}
n_{2}+1 \\
2
\end{array}\right)
$$

Moreover, the height and the relative height of each peak are increased by one, so $n_{j}$ now counts the peaks whose relative height is at least $j$ and for which the difference of the height and the relative height is at most $k-2 j-1$.

We then insert the minimal path starting at $(0,0)$ with $n_{1}-n_{2}$ NES peaks of height one at the beginning of our path. The sum of the abscissas of these new peaks is $\left(\begin{array}{c}n_{1}-n_{2}+1 \\ 2\end{array}\right)$ and they increase the abscissa of each of the old peaks by $n_{1}-n_{2}$. Note that none of the new peaks are counted by $n_{2}$ and that $n_{1}$ now counts the total number of peaks. Altogether, the two operations introduce a factor

$$
\left.a^{n_{2}} q_{2}^{\left(\begin{array}{c}
n_{2}+1 \\
2
\end{array}\right)} \times a^{n_{1}-n_{2}} q^{\left(\begin{array}{c}
n_{1}-n_{2}+1 \\
2
\end{array}\right)+n_{2}\left(n_{1}-n_{2}\right)}=a^{n_{1}} q_{2}^{\left(n_{2}+1\right.}\right) .
$$

The factor $(-1 / a)_{n_{1}}$ corresponds to a partition into distinct parts which lie in $\left[0, n_{1}-1\right]$. If this partition contains a part $j-1\left(1 \leq j \leq n_{1}\right)$, we transform the $j$ th NES peak from the right into a NESE peak. This operation increases the major index of the path by $j-1$.

The factor $(q)_{n_{1}-n_{2}}^{-1}$ corresponds to a partition $\left(b_{1}, b_{2}, \ldots, b_{n_{1}-n_{2}}\right)$ where $b_{1} \geq b_{2} \geq \ldots \geq b_{n_{1}-n_{2}} \geq$ 0 . For $1 \leq j \leq n_{1}-n_{2}$, the $j$ th of the peaks we inserted before (counted from the right) is moved to the right $b_{j}$ times according to the following rules. The first set of rules is described by Figure 2 . Note that rules 2 and 5 can only be applied if the height of the peak being moved is at most $k-3$, since otherwise they would create a valley of height greater than $k-3$.

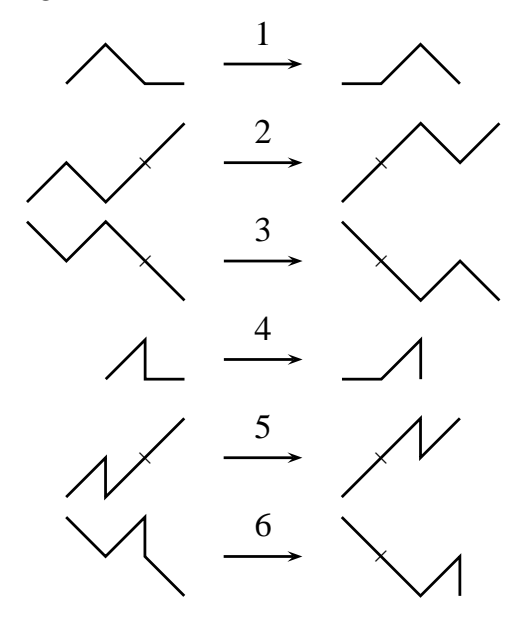

Fig. 2: The first set of rules for moving peaks.

The second set of rules is meant to avoid creating valleys of height greater than $k-3$. See (4, pp. 220-222) for details. 
It can be shown that the peaks we move are still not counted by $n_{2}$ after being moved and that the construction procedure is uniquely reversible.

Proof of Theorem 1.4: It uses the same methods as the proof of the previous theorem. We treat the case $r=1$ first. $W_{2,0}(a, q)$ is the same as $V_{2,0}(a, q)$ except for an additional factor $q^{n}$ which inserts an East step in front of the first peak and a factor $\left(1-q^{2 n+1}\right)^{-1}$, corresponding to a partition $\nu$ into parts equal to $2 n+1$, which we encode by inserting at the beginning of the path a mountain whose height is the number of parts of $\nu$. Here, $n$ is not the number of peaks but the number of plains (a plain is an inclusion-maximal sequence of consecutive horizontal steps).

For $k=2$ and $t=1$, we have an additional factor $q^{n}$ which adds a South-East step at the beginning of the path, from $(0,1)$ to $(1,0)$.

For $k$ odd, the inductive step is based on the following lemma:

Lemma $2.3((4))$

$$
\frac{q^{n_{1}^{2}+\cdots+n_{r}^{2}+n_{1}+\cdots+n_{k-t-1}+2 n_{k-t}+\cdots+2 n_{r}}}{(q)_{n_{1}-n_{2}} \cdots(q)_{n_{r-1}-n_{r}}(q)_{n_{r}}\left(q ; q^{2}\right)_{n_{r+1}}}
$$

is the generating function for paths with no South steps starting at $(0, t)$, with no valleys above height $k-3$, and such that for $1 \leq j \leq r$, there are exactly $n_{j}+1$ peaks whose relative height is at least $j$ and for which the difference between the height and the relative height is at most $k-2 j-1$.

Let $k^{\prime}=k-2, t^{\prime}=t-1$ if $t \leq k-2, t^{\prime}=k-3$ if $t=k-1$. We consider a path with no South steps starting at $\left(0, t^{\prime}\right)$, with no valleys above height $k^{\prime}-3=k-5$, and such that for $2 \leq j \leq r$ there are exactly $n_{j}+1$ peaks whose relative height is at least $j-1$ and for which the difference between the height and the relative height is at most $k^{\prime}-2(j-1)-1=k-2 j-1$.

If $t \leq k-2$, we need to introduce the factor $(-1 / a)_{n_{1}} a^{n_{1}} q^{\left({ }^{n_{1}+1}\right)+n_{1}} /(q)_{n_{1}-n_{2}}$. We do the volcanic uplift and move peaks as before; the only difference is that the path we insert starts at $(0, t)$ and ends at $\left(n_{1}-n_{2}, t-1\right)$ : there is one NESE peak at $(0, t)$ followed by $n_{1}-n_{2}-1$ NES peaks of height one.

If $t=k-1$, we need to introduce the factor $\left.(-1 / a)_{n_{1}} a^{n_{1}} q^{\left(n_{1}+1\right.}\right)+n_{1} /(q)_{n_{1}-n_{2}}$. In this case, the path we insert starts at $(0, k-1)$, passes through $(2, k-3)$ and ends at $\left(n_{1}-n_{2}+1, k-3\right)$ : there is now one NESE peak at $(0, k-1)$ followed by $n_{1}-n_{2}-1$ NES peaks of height $k-3$.

For $k$ even, the proof is very similar.

\section{$3 n$-color overpartitions}

The results on $n$-color overpartitions are essentially reformulations of those on lattice paths. Indeed, we can associate to a lattice path counted by $V_{k, t}$ or $W_{k, t}$ an $(n+t)$-color overpartition in the following way: a peak $(x, y)$ is encoded by a part $x_{y}$, which is overlined if and only if the corresponding peak is a NES peak.

The weighted difference of two parts $m_{i}$ and $n_{j}$ in an $n$-color overpartition, denoted $\left(\left(m_{i}-n_{j}\right)\right)$, is defined to be $m-i-n-j$. We will now show how the conditions on the lattice paths translate to weighted difference conditions on the $n$-color overpartitions. Let us first consider the case of two consecutive peaks $(m, i)$ and $(n, j)$ with $m \geq n$ separated by a valley (which is equivalent to the fact that $\left(\left(m_{i}-n_{j}\right)\right)+\chi\left(n_{j}\right.$ overlined $\left.) \leq 0\right)$. 
The first condition is independent of $k$ and is illustrated by Figure 3 . If $i \leq j$, we have $m-n \geq j-i+$ $2-\chi((n, j)$ is a NES peak $) \geq i-j+2-\chi((n, j)$ is a NES peak $)$, so $\left(\left(m_{i}-n_{j}\right)\right)+\chi\left(n_{j}\right.$ overlined $) \geq$ $-2(j-1)$ and $\left(\left(m_{i}-n_{j}\right)\right)+\chi\left(n_{j}\right.$ overlined $) \geq-2(i-1)$. Altogether, we have $\left(\left(m_{i}-n_{j}\right)\right)+$ $\chi\left(n_{j}\right.$ overlined $) \geq-2 \min (i-1, j-1)$. The case $i \geq j$ is analogous. Note that it also rather easy to see, considering the different cases $\left(i \leq j\right.$ or $i \geq j$ and $n_{j}$ overlined or not) that $\left(\left(m_{i}-n_{j}\right)\right)+\chi\left(n_{j}\right.$ overlined) is always even if it is nonpositive, as required by Theorem 1.1.

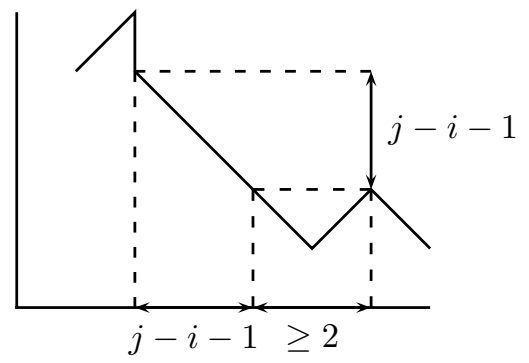

Fig. 3: Illustration of the first weighted difference condition for $i \leq j$, in the case where $(n, j)$ is a NES peak.

The second condition is illustrated by Figure 4. Since the valley between our two peaks has height at most $k-3$, we have $m-n \geq i+j-2 k+6-\chi((n, j)$ is a NES peak), so for the $n$-color overpartition, we have $\left(\left(m_{i}-n_{j}\right)\right)+\chi\left(n_{j}\right.$ overlined $) \geq-2(k-3)$. Putting together the two conditions, we get $\left(\left(m_{i}-n_{j}\right)\right)+\chi\left(n_{j}\right.$ overlined $) \geq-2 \min (i-1, j-1, k-3)$.

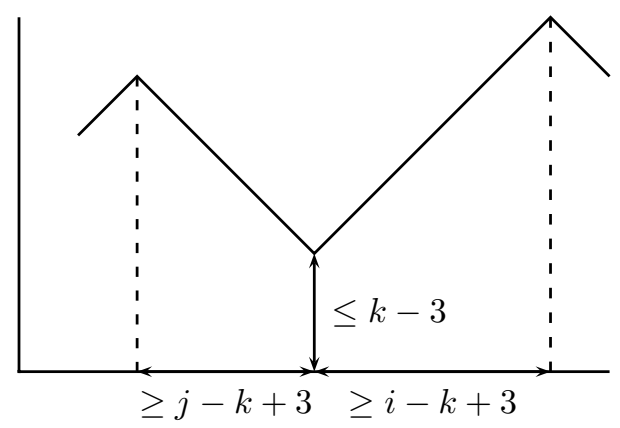

Fig. 4: Illustration of the second weighted difference condition in the case where $(n, j)$ is not a NES peak.

For non-consecutive peaks, we remark that if the South steps between our two peaks were changed into South-East steps, we would come down to the case of $n$-color partitions, where we have that if $\left(\left(m_{i}-n_{j}\right)\right)$ is nonpositive, then it is even and greater than or equal to $-2 \min (i-1, j-1, k-3)$. Such a change would increase $m$ by the number of South steps between our two peaks, which is the number of NES peaks between $(n, j)$ included and $(m, i)$ excluded, or, in the corresponding $n$-color overpartition, the number of overlined parts whose size lies in the interval $[n, m)$. This explains condition (i) of Theorem 1.1 . 
Moreover, if $t \geq 1$, the condition on the starting height translates to the condition that there is a part of the form $x_{x+t}$ : if the path begins with a South-East step (resp. with a South step), it will be a part $0_{t}$ $\left(\operatorname{resp} \overline{0}_{t}\right.$ ), and if the path begins with a North-East step, it will be a part $x_{x+t}$ with $x \geq 1$. There is no such condition if $t=0$ since the path can start with a North-East step or with an East step. The extra condition of Theorem 1.4 also clearly accounts for that of Theorem 1.2: to the NES peak $(x, x+t)$, we associate a non-overlined part $x_{x+t}$.

\section{Infinite products}

To prove Theorem 1.5, we will use the Bailey lattice structure from (3). Recall that a pair of sequences $\left(\alpha_{n}, \beta_{n}\right)$ form a Bailey pair with respect to $a$ if for all $n \geq 0$ we have

$$
\beta_{n}=\sum_{r=0}^{n} \frac{\alpha_{r}}{(q)_{n-r}(a q)_{n+r}}
$$

We need the following lemma which is a special case of Theorem 3.1 of (3):

Lemma 4.1 If $\left(\alpha_{n}, \beta_{n}\right)$ is a Bailey pair with respect to 1 , then we have

$$
\begin{aligned}
\frac{1}{(q,-a q)_{\infty}} \sum_{n_{1} \geq \cdots n_{r} \geq 0} & \frac{(-1 / a)_{n_{1}} a^{n_{1}} q^{\left(\begin{array}{c}
n_{1}+1 \\
2
\end{array}\right)+n_{2}^{2}+\cdots+n_{r}^{2}}}{(q)_{n_{1}-n_{2}} \cdots(q)_{n_{r-1}-n_{r}}} \beta_{n_{r}} \\
=\frac{\alpha_{0}}{(q)_{\infty}^{2}}+\frac{1}{(q)_{\infty}^{2}} \sum_{n \geq 1} & \left(\frac{(-1 / a)_{n} a^{n} q^{r n+(r-1)\left(n^{2}-n\right)+\left(\begin{array}{c}
n \\
2
\end{array}\right)}}{(-a q)_{n}\left(1-q^{2 n}\right)} \alpha_{n}\right. \\
- & \left.\frac{(-1 / a)_{n-1} a^{n-1} q^{r(n-1)+(r-1)\left((n-1)^{2}-(n-1)\right)+\left(\begin{array}{c}
n-1 \\
2
\end{array}\right)+2 n-2}}{(-a q)_{n-1}\left(1-q^{2 n-2}\right)} \alpha_{n-1}\right) .
\end{aligned}
$$

Proof: We set $i=0, a=1, \rho_{1}=-1 / a$, and let $n$ as well as all remaining $\rho_{j}$ and $\sigma_{j}$ tend to infinity in Theorem 3.1 of (3) to get this result.

We first prove (1.5) for $k$ odd. Setting $a=1$ in Lemma 4.1 and using the Bailey pair with respect to 1 (3, equation (5.7), corrected)

$$
\alpha_{n}=\left\{\begin{array}{ll}
0 & \text { if } n \text { odd } \\
(-1)^{\frac{n}{2}} q^{\frac{3 n^{2}-2 n}{4}}\left(1+q^{n}\right) & \text { otherwise }
\end{array} \quad \text { and } \quad \beta_{n}=\frac{1}{(q)_{n}\left(q ; q^{2}\right)_{n}}\right.
$$


we obtain

$$
\begin{aligned}
& \sum_{n_{1} \geq \cdots n_{r} \geq 0} \frac{(-1 / a)_{n_{1}} a^{n_{1}} q^{\left(\begin{array}{c}
n_{1}+1 \\
2
\end{array}\right)+n_{2}^{2}+\cdots+n_{r}^{2}}}{(q)_{n_{1}-n_{2}} \cdots(q)_{n_{r-1}-n_{r}}(q)_{n_{r}}\left(q ; q^{2}\right)_{n_{r}}} \\
& =\frac{(-q)_{\infty}}{(q)_{\infty}}\left(1+\sum_{n \geq 2}\left(\frac{2}{1+q^{n}} \frac{q^{\left(r-\frac{1}{2}\right) n^{2}+\frac{n}{2}}}{1-q^{2 n}} \alpha_{n}-\frac{2}{1+q^{n-1}} \frac{q^{\left(r-\frac{1}{2}\right)(n-1)^{2}+\frac{n-1}{2}}}{1-q^{2(n-1)}} \alpha_{n-1}\right)\right) \\
& =\frac{(-q)_{\infty}}{(q)_{\infty}}\left(1+\sum_{n \geq 1} \frac{2}{1+q^{2 n}} \frac{q^{(4 r-2) n^{2}+n}\left(1-q^{4 n}\right)}{1-q^{4 n}}(-1)^{n} q^{3 n^{2}-n}\left(1+q^{2 n}\right)\right) \\
& =\frac{(-q)_{\infty}}{(q)_{\infty}}\left(1+2 \sum_{n \geq 1} q^{(4 r+1) n^{2}}(-1)^{n}\right) \\
& =\frac{(-q)_{\infty}}{(q)_{\infty}} \sum_{n=-\infty}^{\infty}\left(q^{4 r+1}\right)^{n^{2}}(-1)^{n} \\
& =\frac{(-q)_{\infty}}{(q)_{\infty}}\left(q^{8 r+2} ; q^{8 r+2}\right)_{\infty}\left(q^{4 r+1} ; q^{8 r+2}\right)_{\infty}\left(q^{4 r+1} ; q^{8 r+2}\right)_{\infty}
\end{aligned}
$$

by Jacobi's triple product identity: $\sum_{n \in \mathbb{Z}} z^{n} q^{n^{2}}=\left(-z q,-q / z, q^{2} ; q^{2}\right)_{\infty}$

$$
\begin{aligned}
& =\frac{(-q)_{\infty}}{(q)_{\infty}} \frac{\left(q^{4 r+1} ; q^{4 r+1}\right)_{\infty}}{\left(-q^{4 r+1} ; q^{4 r+1}\right)_{\infty}} \\
& =\frac{(-q)_{\infty}}{(q)_{\infty}} \frac{\left(q^{2 k-1} ; q^{2 k-1}\right)_{\infty}}{\left(-q^{2 k-1} ; q^{2 k-1}\right)_{\infty}}
\end{aligned}
$$

If $k$ is even, the proof is identical except that we use the following Bailey pair (3, equation (5.8), corrected):

$$
\alpha_{n}=\left\{\begin{array}{ll}
0 & \text { if } n \text { odd } \\
(-1)^{\frac{n}{2}} q^{\frac{n^{2}-2 n}{4}}\left(1+q^{n}\right) & \text { otherwise }
\end{array} \quad \text { and } \quad \beta_{n}=\frac{q^{\left(\begin{array}{c}
n \\
2
\end{array}\right)}}{(q)_{n}\left(q ; q^{2}\right)_{n}}\right.
$$

Equation (1.6) is proved using the same Bailey pairs, setting $(a, q)=\left(q^{-1}, q^{2}\right)$ in Lemma 4.1.

For (1.7), we use the following lemma: 
Lemma 4.2 If $\left(\alpha_{n}, \beta_{n}\right)$ is a Bailey pair with respect to $q^{2}$, then for all $k-r-1 \leq t \leq k-1$, we have

$$
\begin{gathered}
\frac{1}{\left(q,-a q^{2}\right)_{\infty}} \sum_{n_{1} \geq \cdots n_{r} \geq 0} \frac{(-1 / a)_{n_{1}} a^{n_{1}} q^{\left(\begin{array}{c}
n_{1}+1 \\
2
\end{array}\right)+n_{2}^{2}+\cdots+n_{r}^{2}+n_{1}+\cdots+n_{r}+n_{k-t}+\cdots+n_{r}}}{(q)_{n_{1}-n_{2}} \cdots(q)_{n_{r-1}-n_{r}}} \beta_{n_{r}} \\
=\frac{\alpha_{0}}{(q)_{\infty}\left(q^{2}\right)_{\infty}}+\frac{1}{(q)_{\infty}\left(q^{2}\right)_{\infty}} \sum_{n \geq 1} q^{(k-t-2)\left(n^{2}-n\right)+\left(\begin{array}{c}
n \\
2
\end{array}\right)+2(k-t-1) n}\left(1-q^{2}\right)\left(\frac{q^{(t+1) n^{2}+2(t+1) n}}{1-q^{2 n+2}} \alpha_{n}\right. \\
\left.-\frac{q^{(t+1)(n-1)^{2}+2(t+1)(n-1)+2 n}}{1-q^{2 n}} \alpha_{n-1}\right) .
\end{gathered}
$$

Proof: We set $t=k-i-1, a=q^{2}, \rho_{1}=-1 / a$, and let $n$ as well as all remaining $\rho_{j}$ and $\sigma_{j}$ tend to infinity in Theorem 3.1 of (3) to get this result.

We also use the following Bailey pairs with respect to $q^{2}$ (3, equations (5.5) and (5.6), corrected):

$$
\alpha_{n}=\left\{\begin{array}{ll}
0 & \text { if } n \text { odd } \\
(-1)^{\frac{n}{2}} q^{\frac{3 n^{2}+2 n}{4}} \frac{1-q^{2 n+2}}{(q)_{2}} & \text { otherwise }
\end{array} \quad \text { and } \quad \beta_{n}=\frac{1}{(q)_{n}\left(q ; q^{2}\right)_{n+1}}\right.
$$

for $k$ odd, and

$$
\alpha_{n}=\left\{\begin{array}{ll}
0 & \text { if } n \text { odd } \\
(-1)^{\frac{n}{2}} q^{\frac{n^{2}-2 n}{4}} \frac{1-q^{2 n+2}}{(q)_{2}} & \text { otherwise }
\end{array} \quad \text { and } \quad \beta_{n}=\frac{q^{\left(\begin{array}{c}
n \\
2
\end{array}\right)}}{(q)_{n}\left(q ; q^{2}\right)_{n+1}}\right.
$$

for $k$ even.

\section{Conclusion}

We showed here how $V_{k, t}(a, q)$ and $W_{k, t}(a, q)$ can be given combinatorial interpretations using $n$-color overpartitions and lattice paths related to overpartitions, just as the series from (3) (the case $a \rightarrow 0$ of our series) were interpreted using $n$-color partitions and lattice paths related to partitions. Specializing our series, we get some identities of the Rogers-Ramanujan type for $n$-color overpartitions. We note that the infinite product in (1.5), which is reminiscent of the first Gordon's theorem for overpartitions (16), appeared in Theorem 1.2 of (17), and that the infinite product in (1.6) is very similar to that in Andrews' generalization of the Göllnitz-Gordon identities (7).

We have obtained more results on these multiple series and other related series, which will be presented in a forthcoming long version of this paper. First, for $a \rightarrow 0$, there exists another combinatorial interpretation of $V_{k, t}$ and $W_{k, t}$ which uses Frobenius partitions with hook difference conditions (2). Our series can be interpreted in the same way using the Frobenius representation of an overpartition $(13 ; 18)$.

Next, if we replace $(q)_{n_{r}}$ by $\left(q^{2} ; q^{2}\right)_{n_{r}}$ in $V_{k, t}$ and $W_{k, t}$, we get two other classes of multiple series which are certainly worth investigating. For $a \rightarrow 0$ and $r=1$, some instances have been studied in (1).

In fact, all those functions can be viewed as a particular case of more general classes of multiple series which should be interpreted in terms of $n$-color overpartition pairs and a more general family of lattice paths introduced in (20) to interpret an extension of the Andrews-Gordon identities to overpartition pairs.

Finally, a question can be raised: is it possible to embed our series in a family of functions with recurrence relations like Andrews's $J_{k, i}(a ; x ; q)$, which are associated to the multiple series from (8)? 


\section{Acknowledgements}

The author wants to thank Jeremy Lovejoy for his help during the preparation of this paper.

\section{References}

[1] A.K. Agarwal, Rogers-Ramanujan identities for $n$-color partitions, J. Number Theory 28 (1988), 299-305.

[2] A.K. Agarwal and G.E. Andrews, Rogers-Ramanujan identities for partitions with " $N$ copies of $N$ ", J. Combin. Theory Ser. A 45 (1987), 40-49.

[3] A.K. Agarwal, G.E. Andrews, and D.M. Bressoud, The Bailey lattice, J. Indian Math. Soc. (N.S.) 51 (1987), 57-73.

[4] A.K. Agarwal and D.M. Bressoud, Lattice paths and multiple basic hypergeometric series, Pacific J. Math. 135 (1989) 209-228.

[5] A.K. Agarwal, Padmavathamma, and M.V. Subbarao, Partition theory, Atma Ram \& Sons, Chandigarh, 2005.

[6] K. Alladi and A. Berkovich, Göllnitz-Gordon partitions with weights and parity conditions, Zeta functions, topology and quantum physics, Dev. Math. 14, 1-17, Springer, New York, 2005.

[7] G.E. Andrews, A generalization of the Göllnitz-Gordon partition theorems, Proc. Amer. Math. Soc. 8 (1967), 945-952.

[8] G.E. Andrews, An analytic generalization of the Rogers-Ramanujan identities for odd moduli, Proc. Nat. Acad. Sci. U.S.A. 71 (1974), 4082-4085.

[9] G.E. Andrews and D.M. Bressoud, On The Burge correspondence between partitions and binary words, Number Theory (Winnipeg, Man., 1983), Rocky Mountain J. Math. 15 (1985), 225-233.

[10] A. Berkovich and P. Paule, Lattice paths, $q$-multinomials and two variants of the Andrews-Gordon identities, Ramanujan J. 5 (2001), 409-425.

[11] D.M. Bressoud, Lattice paths and the Rogers-Ramanujan identities, Number Theory, Madras 1987, 140-172, Lecture Notes in Math. 1395, Springer, Berlin, 1989.

[12] S. Corteel, private communication.

[13] S. Corteel and J. Lovejoy, Overpartitions, Trans. Amer. Math. Soc. 356 (2004), 1623-1635.

[14] S. Corteel and O. Mallet, Overpartitions, lattice paths, and Rogers-Ramanujan identities, J. Combin. Theory Ser. A 114 (2007), 1407-1437.

[15] G. Gasper and M. Rahman, Basic Hypergeometric Series, Cambridge Univ. Press, Cambridge, 1990.

[16] J. Lovejoy, Gordon's theorem for overpartitions. J. Combin. Theory Ser. A 103 (2003), 393-401. 
[17] J. Lovejoy, Overpartition theorems of the Rogers-Ramanujan type, J. London Math. Soc. (2) 69 (2004), 562-574.

[18] J. Lovejoy, Rank and conjugation for the Frobenius representation of an overpartition, Ann. Comb. 9 (2005) 321-334.

[19] J. Lovejoy and O. Mallet, $n$-color overpartitions, twisted divisor functions, and Rogers-Ramanujan identities, submitted.

[20] J. Lovejoy and O. Mallet, Overpartition pairs and two classes of basic hypergeometric series, $A d v$. Math. 217 (2008), 386-418. 
